\title{
QUALIDADE DE VIDA DE MULHERES APÓS HISTERECTOMIA RADICAL
}

\section{QUALITY OF LIFE OF WOMEN AFTER RADICAL HYSTERECTOMY}

\author{
Fabiana Rezer ${ }^{\text {** }}$ Isabel Silva Oliveiral"; Wladimir Rodrigues Faustinol
}

\begin{abstract}
Resumo. O Câncer de Colo de Útero é comum na população feminina, implicando na necessidade de realizar histerectomia, total ou parcial do Útero. Objetivou-se avaliar a qualidade de vida de mulheres que realizaram histerectomia radical após câncer de colo uterino. Trata-se de uma pesquisa descritiva, exploratória e com abordagem quantitativa. O estudo tomou como objeto 15 mulheres que fizeram histerectomia radical decorrente do câncer de colo uterino. Foram coletados dados utilizando o instrumento World Health Organization Quality of Life Group para avaliação da qualidade de vida e, após submissão ao Comitê de Ética e Pesquisa com Seres Humanos, obteve-se a aprovação. Os resultados mostraram que o domínio físico apresentou insatisfação no quesito energia do dia a dia $(2,0)$. O domínio psicológico demonstrou insatisfação na memória, pensar e aprender $(2,1)$. Domínio das relações sociais o quesito menor avaliador foi a atividade sexual $(3,0)$. Domínio do meio ambiente o item menos satisfatório foi o acesso ao serviço de saúde $(1,7)$. Na soma dos domínios percebe-se que o domínio das relações sociais $(3,1)$, seguido do domínio meio ambiente $(3,5)$, apresentaram os piores índices. Já os domínios físicos $(4,1)$ e psicológico $(4,0)$ tiveram as melhores avaliações. Com isso, o estudo aponta que a histerectomia é um evento que repercute na qualidade de vida das mulheres, com atenção especial as relações sociais.
\end{abstract}

PALAVRAS-CHAVE: Histerectomia. Saúde da Mulher. Qualidade de Vida. Cuidados Pós-Operatórios.

Abstract. Cervical Cancer is common in the female population, implying the need to perform a total or partial hysterectomy of the uterus. The objective was to evaluate the quality of life of women who underwent total hysterectomy after cervical cancer. This is a descriptive, exploratory research with a quantitative approach, the research was carried out with 15 women who underwent radical hysterectomy due to cervical cancer. Data were collected using the World Health Organization Quality of Life Group instrument to assess the quality of life. The research was approved by the Ethics and Research Committee with Human Beings. The results showed that the physical domain was dissatisfied in terms of day-to-day energy (2.0). The psychological domain showed dissatisfaction in memory, thinking and learning (2.1). In the domain of social relationships, the lowest evaluator item was sexual activity (3.0). In the domain of the environment, the least satisfactory item was access to health services (1.7). Overall, it was observed that the domain of social relationships (3.1) followed by the domain of the environment (3.5), presented the worst indexes. The physical (4.1) and psychological (4.0) domains presented the best outcomes. Thus, the study points out that a hysterectomy is an event that affects women's quality of life, especially in social relationships.

KEYWORDS: Hysterectomy. Women's Health. Quality of Life. Postoperative Care. 


\section{INTRODUÇÃO}

O câncer de colo de útero é o terceiro tipo de câncer que acomete a população feminina, com elevadas taxas de mortalidade. Em 2020, foram detectados 16.590 casos, com índice de mortalidade em 6.596 óbitos, correspondente a 40\% dos casos. O Instituto Nacional de Câncer (INCA) estima o diagnóstico de 16.590 casos novos de câncer de colo uterino a cada triênio, ocupando a terceira posição no país. ${ }^{1,2}$

O índice de mortalidade vem decaindo com o rastreio precoce e com a possibilidade de tratamento, que deve ser avaliado individualmente. Algumas alternativas envolvem a quimioterapia, radioterapia e a remoção cirúrgica do útero, ou de partes do órgão atingidas. Entre as propostas de tratamento, a Histerectomia é um procedimento realizado para a remoção do útero, essa cirurgia pode ser feita por duas vias, classificando-se em histerectomia abdominal ou vaginal. No Sistema Único de Saúde (SUS) a histerectomia é o segundo maior motivo de cirurgia em mulheres ainda em capacidade reprodutiva. 3,4

O procedimento não pode ser revertido, elevando a preocupação da mulher, após o ato cirúrgico, compreendendo sua expectativa em relação ao universo feminino, relação conjugal, meio familiar, cultural e social, desencadeando fortes reações emocionais e mudanças na autoestima relacionadas à sua imagem corporal. 5,6

Nesse contexto, a histerectomia pode causar em algumas mulheres sentimento de insegurança, ansiedade e impotência. Sabendo que o útero é conhecido como sinônimo de feminilidade, fertilidade e sexualidade, surge então a preocupação em relação a aceitação pelo parceiro e pelos significados sociais concedidos culturalmente às mulheres que foram submetidas ao procedimento, fatores que podem repercutir negativamente na qualidade de vida. ${ }^{7}$

Para além das alterações psicológicas inerentes a cirurgia ginecológica de remoção do útero, ocorrem alterações funcionais e físicas resultantes do procedimento. Com isso, o tratamento cirúrgico pode resultar em reações psicológicas adversas atribuídas ao significado emocional que o útero tem para a mulher. Isso reflete na qualidade de vida, tendo uma repercussão específica. Contudo, tais impactos ainda não estão totalmente claros na literatura, sendo assim, a qualidade de vida de mulheres histerectomizadas é marcada por uma fase de adaptação, como também, de adquirir estabilidade emocional e psicológica, sendo um fator de ansiedade constante no dia a dia da mulher. 8

A expressão Qualidade de Vida (QV) é definida como um conjunto de comportamentos que incluem hábitos, construído por cada indivíduo e, consequentemente, pode ser modificado de forma individual, determinando assim suas escolhas. ${ }^{9}$ As mudanças da imagem corporal e a sensação de estar diferente das outras mulheres implicam em atitudes de rejeição e baixa autoestima. Tal fato se refere a realização pessoal da mulher, podendo gerar um impacto maior no estilo de vida das mulheres que se submetem a cirurgia ainda em idade fértil. ${ }^{7}$

Este trabalho é relevante por 
permitir essencialmente compreender que a histerectomia radical pode desencadear grandes impactos na qualidade de vida da mulher, em conjunto com sua representatividade, identificando alterações físicas, ambientais, psicológicas e sociais.

Assim, visando contribuir na construção do conhecimento dos possíveis fatores que podem influenciar na QV de mulheres que realizaram histerectomia radical, questiona-se: Qual é a qualidade de vida de mulheres após a histerectomia

\section{MATERIAL E MÉTODOS}

Trata-se de um estudo exploratório e com abordagem quantitativa, com amostragem não probabilística, por conveniência. A pesquisa foi realizada com 15 mulheres que realizaram histerectomia radical no setor público, todas eram pertencentes ao município de Guarantã do Norte na Região Norte de Mato Grosso. A população de 100 mulheres que fizeram histerectomia foi identificada pelo cálculo amostral, com erro amostral de $4 \%$, totalizado 15 mulheres como suficientes para compor a amostra.

Teve como critérios de inclusão, mulheres que realizaram histerectomia do tipo radical, até no máximo 02 anos, e excluídas mulheres histerectomizadas com idade inferior a 18 anos e que realizaram o procedimento por outros motivos que não fosse câncer de colo de útero.

Os dados foram coletados através do questionário de qualidade de vida de World Health Organization Quality of Life Group (WHOQOL)-Bref, contendo 26 questões, sendo duas sobre aspectos gerais radical? Quais são os fatores mais afetados na qualidade de vida de mulheres após o procedimento? Por isso, é relevante abordar o assunto proposto, justificando que a alta incidência de câncer de colo uterino e consequente realização da histerectomia radical podem influenciar na QV. O presente estudo objetivou avaliar a qualidade de vida em mulheres que realizaram histerectomia radical e contribuiu para verificar quais são os aspectos mais afetados com o procedimento.

da QV e as demais 24 questões abordaram quatro domínios: 1) domínio físico (dor e desconforto, energia e fadiga, dependência de medicações, mobilidade sono, repouso, atividades diárias e capacidade de trabalho); 2) domínio psicológico (sentimentos positivos e negativos, espiritualidade, crenças, aprendizagem/ memória e concentração, aceitação da imagem corporal e autoestima); 3) relações sociais (relações pessoais, atividade sexual, suporte/apoio social); e 4) meio ambiente (segurança física, ambiente físico, recursos financeiros, novas informações, recreação e lazer, lar, cuidados de saúde e transporte). Questões respondidas individualmente e todos os domínios foram considerados.

A avaliação dos dados foi realizada a partir de uma escala likert de 1 (muito ruim) até 5 pontos (muito bom). Destacase que quanto mais próximo de 5 melhor foi a avaliação da qualidade de vida no respectivo domínio e que os dados foram apresentados em forma de tabelas.

O estudo atendeu as diretrizes 
preconizadas pela Resolução $\mathrm{n}^{\circ} 466$ de 12 de dezembro de 2012 do Comitê de Ética em Pesquisas com Seres Humanos, com CAAE: 35350820.9.0000.5587 e parecer de aprovação $n^{\circ} 4 \cdot 306.578$. Todos os participantes assinaram o Termo de Consentimento Livre e Esclarecido (TCLE).

\section{RESULTADOS}

A amostra incluiu 15 mulheres histerectomizadas. A Tabela 1 apresenta dados sobre a média dos domínios sobre a QV. Para conseguir a média todos os escores foram somados e divididos pelo total de participantes, o domínio que apresenta menor índice relacionado a qualidade de vida é o de relações sociais $(3,1)$, seguido do meio ambiente $(3,5)$ e psicológico $(4,0)$. Em contrapartida, o melhor índice relacionado a qualidade de vida foi considerada no domínio Físico $(4,1)$.

TABELA 1: Domínios sobre a Qualidade de Vida (QV) das mulheres histerectomizadas, segundo WHOQOL-Bref. Região Norte de Mato Grosso, Brasil.

\begin{tabular}{lc}
\hline Domínios & Média dos Escores \\
\hline \hline Relações sociais & 3,1 \\
Meio ambiente & 3,5 \\
Psicológico & 4,0 \\
Físico & 4,1 \\
\hline
\end{tabular}

A Tabela 2 apresenta dados sobre o domínio físico, o item que possui menor índice de satisfação é o: energia no dia a dia, com escore 2,0, sendo considerada
"Muito pouco". O item com melhor índice de satisfação foi o tratamento médico, com escore 4,2.

TABELA 2: Domínio físico das mulheres histerectomizadas, segundo WHOQOL-Bref. Região Norte de Mato Grosso, Brasil.

\begin{tabular}{lcc}
\hline Domínio físico & Escore mínimo & Escore máximo \\
\hline \hline Capacidade de trabalho & 3,5 & 3,7 \\
Sono e repouso & 3,2 & 3,6 \\
Mobilidade & 3,3 & 3,4 \\
Atividade e lazer & 3,1 & 3,5 \\
Energia do dia a dia & 2,0 & 3,5 \\
Tratamento médico & 3,5 & 4,2 \\
Dor física & 3,1 & 3,8 \\
\hline
\end{tabular}

A Tabela 3 apresenta variáveis relacionadas ao domínio psicológico. O item que possui menor satisfação está relacionado a memória, pensar e aprender, 
com escore de 2,1, sendo considerada "muito

de sentimentos negativos, com escore 4,5.

pouco". O item com melhor satisfação foi o

TABELA 3: Domínio psicológico das mulheres histerectomizadas, segundo WHOQOL-Bref. Região Norte de Mato Grosso, Brasil.

\begin{tabular}{lcc}
\hline Domínio Psicológico & Escore mínimo & Escore máximo \\
\hline Sentimentos positivos & 3,5 & 4,1 \\
Memória, pensar e aprender & 2,1 & 4,0 \\
Autoestima & 3,3 & 4,2 \\
Aparência e imagem corporal & 3,5 & 3,7 \\
Relações pessoais, família e apoio & 3,2 & 3,5 \\
Sentimentos negativos & 3,5 & 4,5 \\
\hline
\end{tabular}

A Tabela 4 apresenta as variáveis relacionadas ao domínio das relações socais. O item que possui menor índice de satisfação é a atividade sexual, com escore 3,0, sendo considerado "satisfeito". O item com melhor satisfação foi de relações pessoais, suporte, apoio e amigos com escore 4,5 .

TABELA 4: Domínio relações sociais das mulheres histerectomizadas, segundo WHOQOL-Bref. Região Norte de Mato Grosso, Brasil.

\begin{tabular}{lcc}
\hline Domínio Relações sociais & Escore mínimo & Escore máximo \\
\hline Relações sociais & 3,5 & 4,1 \\
Relações pessoais, suporte, apoio e amigos & 3,5 & 4,5 \\
Atividade sexual & 3,0 & 3,0 \\
\hline
\end{tabular}

A Tabela 5 apresenta variáveis relacionas ao domínio meio ambiente. $\mathrm{O}$ item que possui menor índice de satisfação é o acesso ao serviço de saúde, com escore de
1,7 , sendo considerado "muito ruim". O item com melhor satisfação foi o ambiente físico $(4,6)$.

TABELA 5: Domínio meio ambiente das mulheres histerectomizadas, segundo WHOQOL-Bref. Região Norte de Mato Grosso, Brasil.

\begin{tabular}{lcc}
\hline Domínio Meio ambiente & Escore mínimo & Escore máximo \\
\hline Ambiente físico, polução, ruído e clima & 3,5 & 4,6 \\
Recursos financeiros & 3,5 & 4,5 \\
Disponibilidade de informações & 3,0 & 3,0 \\
Transporte & 2,0 & 3,2 \\
Condições de lar & 3,1 & 3,5 \\
Acesso ao serviço de saúde & 1,7 & 3,2 \\
\hline \hline
\end{tabular}

Fonte: primária, 2021. 


\section{DISCUSSÃO}

As principais alterações encontradas nesse estudo respectivo aos domínios da qualidade de vida com maior comprometimento foram os aspectos das relações sociais no que tange a atividade sexual, e meio ambiente no que se refere ao acesso aos serviços de saúde e transporte.

A insatisfação com a atividade sexual foi um importante indicador das alterações na qualidade de vida, em consonância, um estudo com 645 mulheres residentes em Cartagena ou Barranquilla, no Caribe colombiano, identificou que 152 mulheres (29,1\%) apresentaram severa deterioração da qualidade de vida, e 55,1\% das mulheres histerectomizadas tinham baixo ou nenhum desejo sexual e $29,3 \%$ relataram moderado desejo. ${ }^{10}$

Já uma pesquisa Brasileira transversal, realizada em Florianópolis (SC), demonstrou que após a cirurgia muitas mulheres apresentaram inatividade sexual posterior (43,8\%), além de detectar disfunção sexual em grande parte $(28,1 \%)$ e dispareunia $(32,8 \%)$ nas que mantinham a sexualidade ativa. ${ }^{11}$ Destaca-se que a opinião negativa da mulher sobre sua sexualidade e feminilidade, após a histerectomia, pode interferir na qualidade de vida.

Além disso, a dificuldade de acesso aos serviços de saúde é um fator que compromete a qualidade de vida de muitas mulheres que, muitas vezes, necessitam se deslocar até outras cidades ou regiões para receber o tratamento. Em comparação, um estudo realizado no Amapá, com 41 mulheres histerectomizadas, identificou que $14,6 \%$ das participantes afirmaram estar insatisfeitas com os serviços de saúde. Isso porque a maioria das mulheres não tiveram acesso a cuidados no sistema de saúde e à informação de forma adequada, sendo desconsiderada a possibilidade de decisão sobre a realização da histerectomia, sem dar atenção para o viver da mulher e suas atividades do cotidiano. ${ }^{12}$

O câncer de colo uterino é um dos mais temidos pelas mulheres, pelo trauma psicológico que muitas vezes pode permanecer pela vida toda. Neste estudo, notam-se importantes impactos psicológicos nas relações pessoais, família e apoio. A perda do útero tem um significado que compromete o bem-estar mental, resultando em sentimentos negativos de solidão, medo e angústias. ${ }^{13}$

Estudo, em Vitória da Conquista (BA), detectou que a maioria das mulheres eram casadas, salientando que o total apoio do cônjuge e da rede familiar no processo de enfrentamento na cirurgia é importante na recuperação e na qualidade de vida das pacientes. Assim, diversos aspectos devem ser considerados, desde o diagnóstico até a realização da histerectomia, o que envolvem diferentes pontos de atenção, revelando que a participação do cônjuge desperta segurança diante da doença. ${ }^{14}$

Outro estudo de revisão sobre qualidade de vida, associada ao tratamento em mulheres com câncer de colo uterino, revelou sentimentos de angústia, receio e insegurança, além de prejuízos na vida sexual, pactuando em alterações psicológicas da mulher posterior a histerectomia. ${ }^{15}$

Apesar de os domínios psicológico e físico apresentarem melhores avaliações na qualidade de vida das mulheres histerectomizadas, quando analisadas as médias e o grau de satisfação de cada escore, é evidente que o domínio físico apresentou melhor evidência positiva. Ainda assim, as alterações mais significativas foram 
alterações físicas de mobilidade e pouca energia para realizar as atividades do dia a dia.

Pesquisa semelhante, feita em uma unidade oncológica em Foz do Iguaçu (PR), destacou que a falta de energia no dia a dia é decorrente da necessidade de adaptação após cirurgia, citando a deambulação precoce como efetiva no auxílio das atividades diárias, assim como, a estimulação gradual das atividades rotineiras. ${ }^{16}$ Além disso, as dificuldades das mulheres na volta da rotina, reorganização e volta ao trabalho, podem gerar desconfortos.

Estudo transversal no Hospital do Câncer II e Instituto Nacional do Câncer demonstra que as mulheres com alguma ocupação, após a cirurgia, apresentaram melhores avaliações nas condições físicas se comparado com as desempregadas. ${ }^{17,18}$ É importante destacar que a idade pode impactar negativamente na qualidade de vida de mulheres em fase reprodutiva e que não desenvolvem nenhuma ocupação.

Em uma perspectiva geral, entre as mulheres participantes, 0 item que apresentou menor qualidade de vida foi o de relações sociais (28\%), em contrapartida, a melhor qualidade de vida foi considerada no domínio Físico (64\%). Por outro lado, estudo recente identificou que a pior qualidade de vida envolve os domínios meio ambiente $(58,34 \%)$ e físico $(61,7 \%)$, com resultados contraditórios desta pesquisa. ${ }^{19}$ Tal fato pode ser explicado pelas diferenças regionais e especificidades do tratamento que cada mulher pode apresentar.

Esse panorama revela que oferecer um cuidado baseado na qualidade de vida, com foco nos domínios mais alterados, combinado a avaliação criteriosa das necessidades das mulheres que realizaram histerectomia, ainda é um grande desafio diário para os profissionais de saúde em muitos contextos assistenciais do Brasil.

Reconhece que entre as limitações do estudo, destaca-se o fato de ter sido desenvolvido em um único município, com características locais que restringem os resultados à regionalidade. E também por ter recrutado somente mulheres, após a realização da cirurgia de histerectomia, não avaliando a qualidade de vida antes da remoção do útero. No entanto, os resultados encontrados poderão servir de alerta aos profissionais de saúde e ainda serem utilizados pelos profissionais que atuam na reabilitação de mulheres, após histerectomia, para fomentar uma melhora na qualidade de vida.

\section{CONCLUSÃO}

O presente estudo permitiu verificar que as mulheres que realizaram histerectomia total apresentaram alterações negativas na qualidade de vida, especialmente nos quesitos relacionados ao meio ambiente $\mathrm{e}$ relações sociais. No entanto, em relações aos domínios psicológicos e físicos foram identificados melhores escores na avaliação.

Percebe-se que as repercussões da histerectomia na vida das mulheres variam de acordo com sua especificidade, levando em consideração o modo de se relacionar, processo de apoio afetivo ou social e informações, sendo significativos para garantir a melhoria dos aspectos psicossociais.

Este estudo poderá contribuir para verificar as principais alterações na qualidade de vida de mulheres histerectomizadas e possibilitar intervenções nos fatores mais afetados da qualidade de vida, bem como 
atender as mulheres de acordo com suas necessidades de forma singular, trazendo a intervenção psicossocial, programas de

\section{REFERÊNCIAS BIBLIOGRÁFICAS}

1. Instituto Nacional do Câncer José Alencar Gomes da Silva [homepage da internet]. Tipos de câncer [acesso em 20 de abril de 2021]. Rio de Janeiro: INCA; 2020. Disponível em: https://www.inca.gov.br

2. Carvalho HCM, Lemos MF. As consequências da histerectomia na sexualidade feminina. Perspectivas em Psicologia. 2021; 21(1): 209-24. Disponível em: https://doi.org/10.14393/PPv21n1a2017-13

3. Barbosa ARS, Santos NA, Rodrigues TS. Experiência de mulheres que realizaram histerectomia: revisão integrativa. Rev. Uningá. 2018; 55(2): 227-41. Disponível em: http://revista.uninga.br/index.php/ uninga/article/view/2202

4. Adorno SS, Mesquista JV, Camac LAL, Silva FGN, Saback MC. Vantagens da histerectomia vaginal comparado à histerectomia abdominal em mulheres sem prolapso genital. Rev. Eletrônica acervo saúde. 2018; supl.17(e97): 1-7. Disponível em: https://doi. org/10.25248/reas.e97.2019

5. Schmidt A, Sehnem GD, Cardoso LS, Quadros JS, Ribeiro AC, Neves ET. Experiência de mulheres histerectomizadas acerca da sexualidade. Esc. Anna Nery. 2019; 23 (4): e20190065. Disponível em: https:// doi.org/10.1590/2177-9465-EAN-2019-0065

6. Salimena AMO, Ribeiro MOS. Significado da histerectomia para a mulher e suas implicações na assistência de enfermagem. Rev. Enferm Bras 2019; 18(3):460-66. Disponível em: https://doi.org/10.33233/ eb.v18i3.2357

7. Siqueira CB, Jayme IS, Campos AAS, Carvalho IGM, Almeida RJ. Repercussões psicossociais da histerectomia em nulíparas em idade fértil. Rev. Eletrônica acervo saúde. 2018; 10(01): 1964-72. promoção à saúde e estímulo a melhoria da qualidade de vida.

Disponível em: http://doi.org/10.25248/REAS347_2018

8. Coelho JCC, Pestana ME, Trevizan FB. Sintomas de ansiedade e depressão em pacientes oncológicos atendidos por equipe de psicologia. Rev. Interciência. 2019; 01(2): 45-52. Disponível em: https://www.fafica. br/revista/index.php/interciencia/article/view/75/18

9. Madeira FB, Filgueira DA, Bosi MLM, Nogueira JAD. Estilos de vida, habitus e promoção da saúde: algumas aproximações. Rev. Saúde Soc. 2018; 27(1): 106-15. Disponível em: https://doi.org/10.1590/S010412902018170520

10. Monterrosa-Castro A, Monterrosa-Blanco A, Beltrán-Barrios T. Insomnia and sexual dysfunction associated with severely worsening quality of life in hysterectomized sexually active women. Rev. leep Sci. 2018; 11(2): 99-105. Available form: http://doi. org/10.5935/1984-0063.20180019

11. Nascimento FC, Deitos J, Luz CM. Comparação da disfunção do assoalho pélvico com função sexual e qualidade de vida em sobreviventes ao câncer ginecológico. Cad. Bras. Ter. Ocup. 2019; 27(3): 62837. Disponível em: https://doi.org/10.4322/2526-8910. ctoAO1640

12. Tostes NCB, Cárdenas AMC, Menezes RAO, Miranda LC, Rocha BC, Inajosa SP. Qualidade de vida e sexualidade de mulheres histerectomizadas em uma maternidade pública da Amazônia Brasileira. Rev. Min. Enferm. 2020 ; 24:e-1292. Disponível em: http://www.dx.doi.org/10.5935/1415-2762.20200021

13. Machado LB, Andres SC, Reginaldo MP, Santos DS, Torres RF. Atuação do enfermeiro na prevenção e detecção do câncer do colo uterino para a melhora de vida de mulheres. Research, Society and 
Development.2021;10(07):e30910716648. Disponível em: https://doi.org/10.33448/rsd-v10i7.16648

14. Tristão FR, Machado MP, Gracia ORZ, Lima DKS. Vivências da mulher frente à histerectomia: aspectos emocionais. Rev. Rede de cuidados a saúde. 2017; 11(01): 01-22. Disponível em: http:// publicacoes.unigranrio.edu.br/index.php/rcs/article/ view/4379/2423

15. Moraes $\sqcup$, Neto AJOM, Menezes JLP, Pereira MGP, Pereira YD, Nobrega VM. Qualidade de Vida Associada ao Tratamento com Radioterapia em Mulheres Acometidas pelo Câncer do Colo do Útero: Revisão Integrativa da Literatura. Rev. Bras. Canc. 2021; 67(3): e-231530. Disponível em: https://doi. org/10.32635/2176-9745.RBC.2021v67n3.1530

16. Cardoso BC, Camargo CR, Fernandes I. Perfil de mulheres submetidas a histerectomia e influência da deambulação na alta hospitalar. Rev. Pleiade. 2017; 11(21): 17-24. Disponível em: https://pleiade. uniamerica.br/index.php/pleiade/article/view/330

17. Monterrosa-Castro A, Monterrosa-Blanco A, Beltrán-Barrios T. Insomnia and sexual dysfunction associated with severely worsening quality of life in hysterectomized sexually active women. Rev. leep Sci. 2018; 11(2): 99-105. Available form: http://doi. org/10.5935/1984-0063.20180019

18. Selvanathan S, Acharya N, Singhal S. Quality of life after hysterectomy and hysteroscopic treatment to preserve the uterus from abnormal uterine bleeding or heavy menstrual bleeding. J Midlife Health. 2019 abril-junho; 10(2): 63-69. Available form: http://doi. org/10.4103/jmh.JMH_15_19

19. Tostes NCB, Cárdenas AMC, Menezes RAO, Miranda LC, Rocha BC, Inajosa SP. Qualidade de vida e sexualidade de mulheres histerectomizadas em uma maternidade pública da Amazônia Brasileira. Rev. Min. Enferm. 2020; 24: e-1292. Disponível em: http://www.dx.doi.org/10.5935/1415-2762.20200021 